\title{
Will Resting Full-Cycle Ratio Substitute Fractional Flow Reserve
}

\author{
M. S. Aditya ${ }^{1}$ \\ ${ }^{1}$ Department of Cardiology, Yashoda Hospital, Secunderabad, India \\ Ind J Car Dis Wom:2020;5:100-101
}

\begin{abstract}
Physiological assessment of coronary artery stenosis added an element of certainty of benefit to the patient undergoing revascularization. Fractional flow reserve (FFR) Keywords

- fractional flow reserve

- instant flow reserve

- resting full-cycle ratio was the gold standard, with good reproducibility, correlation with noninvasive inducible ischemia testing and, most importantly, clinical benefit. Increased usage also brought into focus some limitations with respect to achieving hyperemia, assessment of sequential stenoses, drift, multivessel disease, chronic total occlusion, fidelity and accuracy of measurement and, in some cases, cost of adenosine. This set the background for nonhyperemic testing which eliminates the need for drug administration and simplifies procedure vastly.
\end{abstract}

\section{Introduction}

Physiological assessment of coronary artery stenosis added an element of certainty of benefit to the patient undergoing revascularization. Fractional flow reserve (FFR) was the gold standard, with good reproducibility, correlation with noninvasive inducible ischemia testing and, most importantly, clinical benefit. ${ }^{1}$ Increased usage also brought into focus some limitations with respect to achieving hyperemia, assessment of sequential stenosis, drift, multivessel disease, chronic total occlusion, fidelity and accuracy of measurement and, in some cases, cost of adenosine. This set the background for nonhyperemic testing which eliminates the need for drug administration and simplifies procedure vastly.

Instant flow reserve (iFr) when used for assessment of intermediate stenoses was established to achieve similar primary endpoint compared with FFR in DEFINE-FLAIR AND iFR SWEDEHEART trials, with correlation reaching $80 \%$ with FFR. This along with significant reduction in patient discomfort and cost opened up vistas for more indices or measurements, eliminating the need for hyperemia. ${ }^{2,3}$ iFR usage is sometimes limited by accurate tracking of wave form, and assumptions of flow and resistance at precise periods in diastole.
In comparison, resting full-cycle ratio (RFR) maximal relative pressure difference in the cardiac cycle is completely independent of the ECG and irrespective of systole or diastole, thus being an unbiased physiological assessment of coronary artery stenosis. The VALIDATE-RFR study by Svanerud et al examined the validation with FFR and iFR and found performance parameters matching these ratios and also unmasked interesting higher ratios in cardiac systole in up to $12 \%$ of cases, potentially opening up cases which would have been missed with iFR. ${ }^{4}$ The present study validates RFR with FFR in a group of patient's representative of Indian practice, although in small number. The correlation between FFR and RFR values in this study reinforce the validity of RFR in providing sufficient discrimination power for assessment of coronary stenoses.

In Indian practice, the relatively easier availability of RFR may make it an acceptable and attractive substitute for RFR/FFR. Uniquely, RFR may not need a high-fidelity ECG and pressure wave analysis software which reduces the complexity of nonhyperemic testing. Of course, two cases in this study showed a dramatic discordance, which will remain a cause for concern. It would be useful to follow these cases and learn about their outcomes. Finally, despite the small sample size and even smaller patients requiring
Address for correspondence

Dr. M.S. Aditya, MD, DM, Department of Radio-Diagnosis, Consultant Cardiologist, Yashoda Hospital, Secunderabad, India, (e-mail: aditya.ms11@gmail.com).
DOI https://doi.org/ 10.1055/s-0040-1713943.

published online

July 24, 2020
C2020 Women in Cardiology and Related Sciences
License terms

(우(1) $\Theta \circledast$ 
revascularization, this study sets the tone for future physiological lesion assessment among the Indian population.

\section{Conflicts of Interest}

None declared.

\section{References}

1 Tonino PAL, De Bruyne B, Pijls NHJ, et al. FAME study investigators. fractional flow reserve versus angiography for guiding percutaneous coronary intervention. N Engl J Med 2009; $15 ; 360(3): 213-24$
2 Davies JE, Sen S, Dehbi H-M, et al. Use of the instantaneous wave-free ratio or fractional flow reserve in PCI. N Engl J Med 2017; 376:1824-1834

3 Götberg M, Christiansen EH, Gudmundsdottir IJ, est al. The iFR-SWEDEHEART investigators. N Engl J Med 2017;376:1813182 Lindroos, M.D., et al., for the iFR-SWEDEHEART Investigators

4 Svanerud J, Ahn JM, Jeremias A, et al. Validation of a novel non-hyperaemic index of coronary artery stenosis severity: the resting full-cycle ratio (VALIDATE RFR) study. EuroIntervention 2018;14(7):806-814 